\title{
The Cauchy Problem for Hyperbolic Operators with Triple Involutive Characteristics
}

\author{
By \\ Enrico BERNARDI*
}

\section{§ 0. Introduction, Notations and Main Results}

It is well known that the Cauchy problem in the $C^{\infty}$ category for hyperbolic linear differential operators whose principal symbol has characteristics of varying multiplicities is, in general, not well-posed unless some Levi conditions on the lower order terms are imposed. When the characteristics have order at most three some results have been proved in [6] for operators generalizing the effectively hyperbolic case and in [2] where, on the other hand, an extension of the non effectively hyperbolic case has been dealt with. In these results the operator was assumed to have a localisation at triple characteristic points which factors out as the product of a real linear hyperbolic form and a quadratic form. In this paper we want to state necessary and sufficient conditions for the Cauchy problem to be well posed for a model equation in which this factorization property does not hold anymore, i.e. in which we have genuine triple characteristics. This equation represents the microlocal model of a general class of operators for which in [3] the propagation of Gevrey singularities has been studied. To be precise let us consider in $R^{n+1}$ with coordinates $x=\left(x_{0}, x_{1}, \cdots, x_{n}\right)$ the following linear differential operator:

$$
P(x, D)=D_{0}^{3}-a\left(D_{1}^{2}+D_{2}^{2}\right) D_{0}+b D_{1}^{3}+p_{2}(x, D)+p_{1}(x, D)+p_{0}(x, D) .
$$

Here $D_{\jmath}=(1 / i) \partial_{x_{j}}, j=0,1, \cdots, n ; a, b \in \boldsymbol{R}$. We shall assume that $p_{3}(x, \xi)$ is hyperbolic with respect to $\left(0, e_{0}\right)$, i.e. $(4 / 27) a^{3}-b^{2}>0 . \quad p_{3}$ vanishes exactly of the third order on the involutive submanifold $\Sigma$ of $\dot{T}^{*} \boldsymbol{R}^{n+1}, \Sigma=\left\{(x, \xi) \mid \xi_{0}=\xi_{1}\right.$ $\left.=\xi_{2}=0\right\}$, which has been assumed of codimension 3 for the sake of simplicity. The necessary conditions for well-posedness of Ivrii-Petkov [5] now assert that $p_{2_{1 \Sigma}}=0$, which however is not enough in this case. In fact our result can be stated as follows.

Theorem 1. Let $P$ as in (0.1). Then a necessary and sufficient condition in

Communicated by S. Matsuura, April 18, 1990.

1991 Mathematics Subject Classification: 35L30.

* Dipartimento di Matematica, Università di Bologna, Piazza di Porta S. Donato 5, 40126 dl Porta Bologna, Italia. 
order that the Cauchy problem for $P$ be well posed in $S_{0}=\left\{x \in \mathbb{R}^{n+1} \mid x_{0}<0\right\}$ is that :

$$
p_{21 \Sigma}=0, \quad H_{p_{21 \Sigma}}=0, \quad p_{1 \mid \Sigma}=0 .
$$

We shall prove that $H_{p_{2 \mid \Sigma}}=0$ by first establishing that $H_{p_{2}}\left(0 ; e_{n}\right) \in \Gamma_{p_{3}}^{\sigma}$, the dual cone with respect to $\sigma=d \xi \wedge d x$, the symplectic two form in the cotangent bundle, of the hyperbolicity cone $\Gamma_{p_{3}}$ of $p_{3}$. Without loss of generality we may assume that $p_{2}(x, D)=\left(c_{0} D_{0}+c_{1} D_{1}+c_{2} D_{2}\right) D_{n}$, hence localizing at $\left(0,-e_{n}\right)$ we obtain that $H_{p_{2 \mid \Sigma}}=0$. All necessary conditions are proved as in Hörmander [4] by means of suitable symplectic dilatations and conjugations with phase functions giving rise to algebraic equations of degree 3 whose complex roots are now important. As far as sufficiency is concerned we remark that once (0.2) holds true it is possible to prove energy estimates for $P$ forming as usual $2 i \Im\langle P u, M u\rangle$ where $u \in C_{0}^{\infty}$ and $M$ is a suitable hyperbolic operator with double characteristics to be properly chosen. We also would like to remark that the results proved in [1] can be applied to the operator $P$ satisfying the Levi conditions (0.2), thus giving propagation of $C^{\infty}$ singularities along $\Gamma_{p_{3}}^{\sigma}$ on the leaves of the natural foliation of $\Sigma$.

\section{§1. Necessity}

Let us recall from [4] that if the Cauchy problem for $P$ is well posed in $S_{0}$ then $\forall K$ compact subset of $\boldsymbol{R}^{n+1} \exists \mu>0$ such that $\forall u \in C_{0}^{\infty}(K)$

$$
\|u\|_{(-\mu)} \leqq C\|P u\|_{(\mu)} .
$$

(See e.g. [4] for a definition of the quotient Sobolev norms $\|\cdot\|(\bar{s})$ ). In orde1 to prove that $(0.2)$ is necessary let us construct asymptotic null solutions of $P u=0$ in order to violate (1.1). Therefore starting from $P$ of the form:

$$
P=D_{0}^{3}-a\left(D_{1}^{2}+D_{2}^{2}\right) D_{0}+b D_{1}^{3}+\left(c_{0} D_{0}+c_{1} D_{1}+c_{2} D_{2}\right) D_{n}+k D_{n}
$$

let us perform the symplectic dilation

$$
\left(x_{0}, x_{1}, x_{2}, x^{\prime}, x_{n}\right) \longrightarrow\left(x_{0}, \frac{1}{\rho} x_{1}, \frac{1}{\rho} x_{2}, x^{\prime}, \frac{1}{\rho} x_{n}\right),
$$

$x^{\prime}=\left(x_{3}, \cdots, x_{n-1}\right)$ and conjugate with $E_{\rho}(x)=\exp \left(i \rho^{2} x_{n}+i \rho^{2} x_{1} \xi_{1}+i \rho^{2} x_{2} \xi_{2}+i \rho \varphi(x)\right)$ we have:

$$
\begin{aligned}
\rho^{-3} P_{\rho}= & {\left[\left(D_{0}+\rho \varphi_{0}\right)^{3}-\frac{a}{\rho^{2}}\left(\left(D_{1}+\rho \varphi_{1}+\rho^{2} \xi_{1}\right)^{2}+\left(D_{2}+\rho \varphi_{2}+\rho^{2} \xi_{2}\right)^{2}\right)\left(D_{0}+\rho \varphi_{0}\right)\right.} \\
& +\frac{b}{\rho^{3}}\left(D_{1}+\rho \varphi_{1}+\rho^{2} \xi_{1}\right)^{3}+\left(c_{0}\left(D_{0}+\rho \varphi_{0}\right)+\frac{c_{1}}{\rho}\left(D_{1}+\rho \varphi_{1}+\rho^{2} \xi_{1}\right)\right. \\
& \left.\left.+\frac{c_{2}}{\rho}\left(D_{2}+\rho \varphi_{2}+\rho^{2} \xi_{2}\right)\right)\left(D_{n}+\rho \varphi_{n}+\rho^{2}\right)+k\left(D_{n}+\rho \varphi_{n}+\rho^{2}\right)\right]
\end{aligned}
$$




$$
\begin{aligned}
= & \left\{\varphi_{0}^{3}-a\left(\xi_{1}^{2}+\xi_{2}^{2}\right) \varphi_{0}+b \xi_{1}^{3}+\cdots+c_{0} \varphi_{0}+c_{1} \xi_{1}+c_{2} \xi_{2}\right\} \\
& +\rho^{-1}\left\{3 \varphi_{0}^{2} D_{0}+\frac{3}{i} \varphi_{0} \varphi_{00}-a\left(\xi_{1}^{2}+\xi_{2}^{2}\right) D_{0}-2 a\left(\varphi_{1} \xi_{1}+\varphi_{2} \xi_{2}\right) \varphi_{0}+3 b \varphi_{1} \xi_{1}^{2}\right. \\
& \left.+c_{0} D_{0}+c_{1} \varphi_{1}+c_{2} \varphi_{2}+\left(c_{0} \varphi_{0}+c_{1} \xi_{1}+c_{2} \xi_{2}\right) \varphi_{n}+k\right\}+O\left(\rho^{-2}\right)
\end{aligned}
$$

where by $O\left(\rho^{-2}\right)$ we denote differential operators with coefficients bounded uniformly after multiplication by $\rho^{-2}$. Let us now show that the $c_{j}$ 's are real. Assume the contrary and consider the eikonal equation :

$$
\varphi_{0}^{3}-a\left(\xi_{1}^{2}+\xi_{2}^{2}\right) \varphi_{0}+b \xi_{1}^{3}+\cdots+c_{0} \varphi^{0}+c_{1} \xi_{1}+c_{2} \xi_{2}=0 .
$$

Since $\xi_{1}, \xi_{2} \in \boldsymbol{R},(1.4)$ certainly has a simple root $\zeta$ with $\mathfrak{\jmath} \zeta<0$. Therefore $\varphi(x)=\zeta x_{0}+i\left(x_{1}^{2}+x_{2}^{2}+\cdots+x_{n}^{2}\right)$ solves $(1.4)$ and $\Im \varphi(x) \geqq C|x|^{2}$ in $x_{0} \leqq 0$. Since the transport equations coming from (1.3) are non characteristics, the standard arguments in [4] prove the thesis. Let us now turn to the second part of the proof, i.e. let us show that the Hamilton vector field of the linear form $c_{0} \xi_{0}+$ $c_{1} \xi_{1}+c_{2} \xi_{2}$ belongs to the propagation cone of the principal symbol. Let us turn back to the eikonal equation (1.4). It is of the form $p_{3}\left(\varphi_{0}, \xi_{1}, \xi_{2}\right)+p_{1}\left(\varphi_{0}, \xi_{1}, \xi_{2}\right)$ $=0$ where $p_{j}$ is a homogeneous polynomial of degree $j$ and $p_{3}$ has real roots in $\varphi_{0}$ for every $\xi_{1}, \xi_{2}$. It is easily verified that the equation $\varphi_{0} \rightarrow\left(p_{3}+p_{1}\right)\left(\varphi_{0}, \xi_{1}, \xi_{2}\right)$ $=0$ has real roots in $\varphi_{0} \forall \xi_{1}, \xi_{2}$ iff $\nabla_{\zeta^{\prime}, \xi_{1}, \xi_{2}} p_{1}$ belongs to the euclidean polar of the hyperbolicity cone of $p_{3}$. Arguing as before shows that $\varphi(x)=\zeta x_{0}+i\left(x_{1}^{2}+\right.$ $\left.x_{2}^{2}+\cdots+x_{n}^{2}\right)$ solves $(1.4), \Im \varphi(x) \geqq C|x|^{2}$ and we finally obtain $H_{p_{2}} \in \Gamma_{p_{3}}^{\sigma}$. Since $P$ is differential this eventually yields that $p_{2}$ must vanish of order 2 on $\Sigma$. Therefore let us show that $p_{1}$ has to vanish on $\Sigma$. The operator can now be thought of the form:

$$
P=D_{0}^{3}-a\left(D_{1}^{2}+D_{2}^{2}\right) D_{0}+b D_{1}^{3}+A\left(D_{0}, D_{1}, D_{2}\right)+k D_{n}
$$

where $A$ denotes a quadratic form in its argument. Performing the symplectic dilation $\left(\xi_{0}, \xi_{1}, \xi_{2}, \xi_{3}, \cdots, \xi_{n-1}, \xi_{n}\right) \rightarrow\left(\rho^{N} \xi_{0}, \rho^{N} \xi_{1}, \rho^{N} \xi_{2}, \xi_{3}, \cdots, \xi_{n-1}, \rho^{3 N} \xi_{n}\right)$, together with its dual one, we can localize $P$ in the following way:

$$
P_{\rho}=D_{0}^{3}-a\left(D_{1}^{2}+D_{2}^{2}\right) D_{0}+b D_{1}^{3}+k D_{n}+O\left(\rho^{-N}\right) .
$$

With another symplectic dilation we can finally "eliminate" $D_{1}$ and $D_{2}$ from (1.6) and, after conjugation with $E_{\rho}(x)=e^{i \rho^{3} x_{n}+\rho \varphi(x)}$, we eventually obtain the eikonal equation :

$$
\varphi_{0}^{3}+k=0 .
$$

Hence if $k \neq 0,(1.7)$ certainly admits a complex root $\zeta$ with $\Im \zeta<0$ and again the standard arguments in constructing an asymptotic solution for the equation $P_{\rho} u_{\rho} \sim 0$ apply, thus proving that $(0.2)$ is necessary. 


\section{§2. Sufficiency}

Here we shall prove that $(0.2)$ is sufficient for the well-posedness of the Cauchy problem for $P$, which we may assume to be of the following form:

$$
\begin{aligned}
P= & D_{0}^{3}-a\left(D_{1}^{2}+D_{2}^{2}\right) D_{0}+b D_{1}^{3}+c_{0} D_{0}^{2}+2 c_{1} D_{1} D_{0}+2 c_{2} D_{2} D_{0} \\
& +2 c_{3} D_{1} D_{2}+c_{4} D_{1}^{2}+c_{5} D_{2}^{2}+c_{6} D_{0}+c_{7} D_{1}+c_{8} D_{2}+c_{9} .
\end{aligned}
$$

As a standard multiplier let us choose $M=D_{0}^{2}-(a / 3) D_{1}^{2}$ and compute :

$$
\begin{aligned}
2 i \Im\langle P u, M u\rangle= & 2 i \Im\left\langle\left( D_{0} M-\frac{2 a}{3} D_{1}^{2} D_{0}-a D_{2}^{2} D_{0}+b D_{1}^{3}+c_{0} M\right.\right. \\
& +\left(c_{1}-\frac{a}{3}\right) D_{1}^{2}+c_{5} D_{2}^{2}+2 c_{1} D_{1} D_{0}+2 c_{2} D_{2} D_{0}+2 c_{3} D_{1} D_{2} \\
& \left.\left.+c_{6} D_{0}+c_{7} D_{1}+c_{8} D_{2}+c_{9}\right) u,\left(D_{0}^{2}-\frac{a}{3} D_{2}^{2}\right) u\right\rangle .
\end{aligned}
$$

Let us first assume that the $c_{j}$ 's are real, $j=1, \cdots, 9$. Then,

$$
\begin{aligned}
2 i \Im\langle P u, M u\rangle= & D_{0}\left\{|M u|^{2}+\Re\left(\left[\begin{array}{cc}
2 a^{2} / 9 & -b \\
-b & 2 a / 3
\end{array}\right]\left[\begin{array}{c}
D_{1}^{2} u \\
D_{1} D_{0} u
\end{array}\right],\left[\begin{array}{c}
D_{1}^{2} u \\
D_{1} D_{0} u
\end{array}\right]\right\rangle\right. \\
& +a\left|D_{2} D_{0} u\right|^{2}+\frac{a^{2}}{3}\left|D_{1} D_{2} u\right|^{2}-2\left(c_{4}-\frac{a}{3}\right) \Re\left\langle D_{1}^{2} u, D_{0} u\right\rangle \\
& -2 c_{5} \Re\left\langle D_{2}^{2} u, D_{0} u\right\rangle-2 c_{1}\left\langle D_{1} D_{0} u, D_{0} u\right\rangle-2 c_{2}\left\langle D_{2} D_{0} u, D_{0} u\right\rangle \\
& -\frac{2 a c_{2}}{3} c_{2}\left\langle D_{2} u, D_{1}^{2} u\right\rangle-\frac{2 a c_{2}}{3} c_{1}\left\langle D_{1} u, D_{1}^{2} u\right\rangle \\
& -2 \Re\left\langle 2 c_{3} D_{1} D_{3} u, D_{0} u\right\rangle-c_{6}|u|^{2}-2 \Re c_{7}\left\langle D_{1} u, D_{0} u\right\rangle \\
& \left.-2 \Re c_{8}\left\langle D_{2} u, D_{0} u\right\rangle-2 \Re c_{9}\left\langle u, D_{0} u\right\rangle\right\} \\
= & D_{0}\left\{|M u|^{2}+E+R\right\}
\end{aligned}
$$

where $E$ denotes the positive leading part:

$$
\Re\left\langle\left[\begin{array}{cr}
2 a^{2} / 9 & -b \\
-b & 2 a / 3
\end{array}\right]\left[\begin{array}{c}
D_{1}^{2} u \\
D_{1} D_{0} u
\end{array}\right],\left[\begin{array}{c}
D_{1}^{2} u \\
D_{1} D_{0} u
\end{array}\right]\right\rangle+a\left|D_{2} D_{0} u\right|^{2}+\frac{a^{2}}{3}\left|D_{1} D_{2} u\right|^{2} .
$$

Let us multiply (2.3) by $i e^{-2 \tau x_{0}}$ and integrate for $x_{0}<0$ which gives:

$$
\begin{aligned}
C \int_{-\infty}^{0}|P u|^{2} e^{-2 \tau x_{0}} d x \geqq & \int_{x_{0}=0}\left(|M u|^{2}+E+R\right) d x^{\prime} \\
& +\tau \int_{-\infty}^{0} e^{-2 \tau x_{0}}\left(|M u|^{2}+E+R\right) d x .
\end{aligned}
$$


Let us now recall from [4] the following energy estimate for $M$ :

$$
\begin{aligned}
C \int_{-\infty}^{0}|M u|^{2} e^{-2 \imath x_{0}} d x \geqq & \tau^{2} \int_{-\infty}^{0}\left(\left|D_{0} u\right|^{2}+\left|D_{1} u\right|^{2}\right) e^{-2 \tau x_{0}} d x \\
& +\tau^{4} \int_{-\infty}^{0}\left(|u|^{2}\right) e^{-2 \tau x_{0}} d x .
\end{aligned}
$$

It is now a simple matter to verify that every term in $R$ can be estimated using Cauchy-Schwartz inequality by (2.6) and $E$. This eventually yields that, with $\tau>0$ sufficiently large,

$$
\begin{aligned}
& C \int_{-\infty}^{0}|P u|^{2} e^{-2 \tau x_{0}} d x \\
& \geqq \tau \int_{-\infty}^{0}\left(\left|D_{1} D_{0} u\right|^{2}+\left|D_{1}^{2} u\right|^{2}+\left|D_{2} D_{0} u\right|^{2}+\left|D_{1} D_{2} u\right|^{2}\right) e^{-2 \tau x_{0}} d x \\
& \quad+\tau^{3} \int_{-\infty}^{0}\left(\left|D_{0} u\right|^{2}+\left|D_{1} u\right|^{2}\right) e^{-2 \tau x_{0}} d x+\tau^{5} \int_{-\infty}^{0}\left(|u|^{2}\right) e^{-2 \tau x_{0}} d x .
\end{aligned}
$$

A similar argument holds if the $c_{j}$ 's are complex and from (2.7) it is now straightforward to derive existence and uniqueness results. We also would like to remark that, although our theorem has been proved in the particular case given by (0.1), all the basic features of a more general setting are preserved and in fact the following result could be proved precisely in the same way:

Theorem 2. Let $P=p_{m}+p_{m-1}+p_{m-2}+\cdots$ be a linear differential operator of order $m$ with $C^{\infty}$ coefficients in $\boldsymbol{R}^{n+1}$. Assume that $P$ is hyperbolic with respect to $d x_{0}, p_{m}$ vanishes of order three on a $C^{\infty}$ involutive submanifold $\Sigma \subset T^{*} \boldsymbol{R}^{n+1}$ and that $\forall \rho \in \Sigma$ the localization at $\rho$ of $p_{m}, p_{m, \rho}(\delta z)=\lim _{t \rightarrow 0} p_{m}(\rho+t \delta z)$ is a homogeneous polynomial of degree three which is strictly hyperbolic with respect to $\left(0, e_{0}\right)$. Then a necessary and sufficient condition in order that the Cauchy problem for $P$ be well posed in $S_{0}=\left\{x \in \boldsymbol{R}^{n+1} \mid x_{0}<0\right\}$ is that:

$$
p_{m-1}(\rho)=0, \quad H_{p_{m-1}}(\rho)=0, \quad p_{m-2}(\rho)=0 \quad \forall \rho \in \Sigma .
$$

\section{References}

[1] Bernardi, E., Propagation of singularities for hyperbolic operators with multiple involutive characteristics, Osaka J. Math., 25 (1988), 19-31.

[2] Bernardi, E. and Bove, A., The Cauchy problem for a class of hyperbolic operators with triple characterics, Proceeding of the Japan Academy.

[3] - Propagation of Gevrey singularities for a class of operators with multiple characteristics, I, Duke Math. Journal, to appear.

[4] Hörmander, L., The Cauchy problem for differential equations with double characteristics, J. Analyse Math., 32 (1977), 110-196.

[5] Ivrii, V.Ya. and Petkov, V.M., Necessary conditions for the correctness of the 
Cauchy Problem for non strictly hyperbolic equations, Uspehi Mat. Nauk., 29:5 (1974), 3-70.

[6] Nishitani, T., Une classe d'opérateurs hyperboliques à caractéristiques multiples (1987), Preprint. 